\title{
Prevalence and genotype distribution of HPV infection among 214,715 women from Southern China, 2012-2018: baseline surveys prior to mass HPV vaccination
}

\section{Lipei Luo}

Southern Medical University https://orcid.org/0000-0001-6032-6481

\section{Ping He}

Southern Medical University

Qiaotu Liu

Chenzhou No1 People's Hospital

\section{Yanghua Jiang}

chenzhou NO.1 People's Hospital

\section{Yang-nan Zhang}

Chenzhou N0.1 people's hospital

\section{Qingzhao Li}

chenzhou N0.1 people's hospital

\section{Qiu Li}

chenzhou N0.1 people's hospital

\section{Shengtao Li}

chenzhou N0.1 people's hospital

\section{Fan Yang}

chenzhou N0.1 people's hospital

\section{Hua Ling}

chenzhou No.1 people's hospital

\section{Xingui Dai}

chenzhou No.1 people's hospital

Zhongyu Li ( $\sim$ lzhy1023@hotmail.com )

university of south china

Hongliang Chen ( $\sim$ chenhongliang2007@126.com )

Southern Medical University and University of South China https://orcid.org/0000-0003-2899-3545

Research article 
Keywords: Human papillomavirus, high-risk HPV, low-risk HPV, prevalence, genotype, single HPV infection, multiple HPV infection

Posted Date: April 6th, 2020

DOI: https://doi.org/10.21203/rs.3.rs-20658/v1

License: (c) (1) This work is licensed under a Creative Commons Attribution 4.0 International License. Read Full License

Version of Record: A version of this preprint was published at BMC Infectious Diseases on April 7th, 2021. See the published version at https://doi.org/10.1186/s12879-021-06019-5. 


\section{Abstract}

Background: The epidemiology on the human papillomavirus (HPV) among females in Southern China is not well-established. Baseline data on the prevalence of HPV infection in China prior to mass prophylactic HPV vaccination would be useful. Thus, this study aimed to determine the type-specific HPV prevalence and distribution among females from Southern China prior to mass HPV vaccination.

Methods : A retrospective cross-sectional study employing 214,715 women attending ChenZhou N0.1 People's Hospital for cervical screening during 2012-2018 was conducted prior to widespread HPV vaccination. HPV genotype was detected using nucleic acid molecular diversion hybridization tests. The overall prevalence, age-specific prevalence, type distribution囚and annual trend was analyzed.

Results : The overall HPV prevalence was 18.71\% (95\% confidence interval [Cl], 18.55\%-18.88\%) among Southern China females. During 2012-2018, the prevalence of HPV infection showed a downward tendency, from $21.63 \%$ (95\% Cl, 21.07\%-22.20\%) in 2012 to $18.75 \%(95 \% \mathrm{Cl}, 18.35 \%-19.16 \%)$ in 2018. Age-specific HPV distribution displayed a peak at young women aged less than 21 years $(33.11 \%, 95 \% \mathrm{Cl}$, $31.13 \%-35.15 \%), 20.07 \%$ ( $95 \% \mathrm{Cl}, 19.70 \%-20.44 \%$ ) among women aged $21-30$ years, $17.29 \%(95 \% \mathrm{Cl}$, $17.01 \%-17.57 \%)$ among women aged $31-40$ years, $17.23 \%(95 \% \mathrm{Cl}, 16.95 \%-17.51 \%)$ among women aged 41-50 years, $21.65 \%(95 \% \mathrm{Cl}, 21.11 \%-22.20 \%)$ among women aged $51-60$ years, and $25.95 \%(95 \% \mathrm{Cl}$, $24.86 \%-27.07 \%$ ) among women aged over 60 years. Of the 21 subtypes identified, the most three prevalent high-risk HPV (HR-HPV) genotypes were HPV52 (5.12\%; 95\% Cl, 21.11\%-22.20\%), -16 (2.96\%; $95 \% \mathrm{Cl}, 2.89 \%-3.03 \%)$, and -58 (2.51\%; $95 \% \mathrm{Cl}, 2.44 \%-2.58 \%)$; the predominant low-risk HPV (LR-HPV) genotypes were HPV81 $(1.86 \% ; 95 \% \mathrm{Cl}, 1.80 \%-1.92 \%)$ and $-6(0.69 \% ; 95 \% \mathrm{Cl}, 0.66 \%-0.73 \%)$ respectively. Incidence of HR-HPV only, LR-HPV only and mixed LR- and HR-HPV were $15.17 \%, 2.07 \%$ and $1.47 \%$ respectively. Besides, single HPV infection accounted for $77.30 \%$ of all positive cases in this study.

Conclusions: This study highlights 1) a high prevalence of HPV infection among females with a decreasing tendency towards 2012-2018, especially for young women under the age of 21 prior to mass HPV vaccination; 2) HPV52, -16 and -58 were the predominant HPV genotypes, suggesting potential use of HPV vaccine covering these HPV genotypes in Southern China.

\section{Background}

Human papillomavirus (HPV) is the leading cause of cervical cancer and responsible for about $91 \%$ of anal cancers, $75 \%$ of vaginal cancers and $70 \%$ oropharynx cancer [1]. It is estimated that there were over 528,000 new cervical cancer patients and 266,000 death worldwide recorded in 2012, among which $85 \%$ occurred in developing countries [2]. In China, 130,000 new cases of cervical cancer are diagnosed annually, especially in young women within the first few years after sexual debuts [3,4]. The morbidity and mortality of cervical cancer incidence in developed countries has fallen over the last two decades because of an enhanced awareness of cervical cancer prevention and effective screening [5]. However, 
infection rate of HPV has fluctuated and not shown a downward trend, indicating a relatively heavy burden of HPV infection in China [6, 7].

HPV genotypes are linked to the degree of cervical lesions [6]. Infections with low-risk HPV (LR-HPV) types, such as HPV6 and -11, cause benign or low-grade changes in cervix cells, genital warts, and recurrent respiratory papillomatosis. Contrarily, high-risk HPV (HR-HPV) type cause cervical, anal, and other genital cancers, which could be detected in $99 \%$ of cervical cancers-the second most common cancer in women worldwide. Especially, HPV16 and - 18 have been found to be the most pathogenic of the HR-HPV types, causing about $70 \%$ of cervical cancers worldwide [7-10]. Of note, most HPV infections are completely asymptomatic, resulting in a delay in diagnosis and follow-up treatment with disastrous consequences [11]. Moreover, HPV-infected individuals without early intervention will develop long-lasting HPV infections that put them at risk for cervical cancer, and increase the risk of sexual transmission to their partners, resulting in approximately estimated $\$ 1.7$ billion in direct medical costs annually in the United States [12].

The prevalence of HPV varies geographically widely, ranging from $6 \%$ in southeastern Asia to $32 \%$ in eastern Africa, and from 6.7-44.5\% in China [13]. HPV screening and vaccine are considered being the most effective measures for preventing HPV infection. HPV vaccine has been clinically applied for more than 10 years [14], however, officially launched on July 2017 in mainland China and available mainly in the developed regions in China currently, such as Beijing, Shanghai, and Guangzhou. Besides, the HPV vaccine made in China was approved in 2020. Representative data on type-specific prevalence of HPV infection in China could provide a baseline estimate to measure the burden of HPV infection and could help guide programs on HPV-based cervical cancer screening and strategies on vaccine-based HPV prevention. To date, the existing data about epidemiological characteristics of HPV infection in Southern China are still scantly, especially lacking large sample study on the genotype prevalence of HPV. Therefore, this paper was conducted to determine the prevalence and type distribution of HPV among 214,715 females in Southern China, including its trends from 2012 to 2018 and age-specific prevalence. Such data provide a baseline pre-vaccine population-based prevalence of HPV in Southern China, and help guide models evaluating impact and cost-effectiveness by comparison between future vaccinated populations and implementation of the prophylactic HPV vaccine according to the specific types' distribution in the region.

\section{Methods}

\section{Study population}

From January 2012 to December 2018, a population of 214,715 females (age range from 19 to 83 years) was enrolled in this study. All participants were from gynecology department and physical examination center of Chenzhou NO.1 People's Hospital. The inclusion criteria are female, HPV-unvaccinated, not being pregnant, and no parturition when sampling.

\section{Cervical specimen collection and management}


Cervical samples were taken by the physician using a $200 \mathrm{~mm}$ polyethylene Cervix brush device (Hybribio Corp, Guangdong) following the regular procedures for speculum examination. Swabs The samples were transferred to a sampling tube containing a transport medium (Hybribio Corp, Guangdong) and stored at 2-8 ${ }^{\circ} \mathrm{C}$ until HPV DNA extraction within a week. Cervical samples can be kept in a transport medium for 2 weeks at $4{ }^{\circ} \mathrm{C}$ according to the manufacturer's manual.

\section{HPV genotyping}

DNA was extracted by Cell Lysis Kit with the negative and positive quality control products throughout the whole process. HPV genotyping was performed using HPV assay kit (Hybribio Limited. Chaozhou, China) for $21 \mathrm{HPV}$ genotype tests via gene amplification technology and diversion hybridization, and the low limit of detection (LOD) for HPV DNA is 500 copies/ml according to manufacturer's protocol. This assay uses HPV L1 consensus PCR primers (MY09/11) for the amplification and a human housekeeping gene $\beta$-globin as an endogenous internal control to ensure appropriate DNA purification, PCR reaction and specimen quality. PCR was carried out in $25 \mu \mathrm{l}$ reaction mixture in a thermal cycler, the cycling parameters of which were as follows: $20^{\circ} \mathrm{C}$ for $10 \mathrm{~min}, 95^{\circ} \mathrm{C}$ for $9 \mathrm{~min}$, followed by 40 cycles $\left(20 \mathrm{~s}\right.$ at $95^{\circ} \mathrm{C}, 30 \mathrm{~s}$ at $55^{\circ} \mathrm{C}$ and $30 \mathrm{~s}$ at $72{ }^{\circ} \mathrm{C}$ ), with a final extension at $72^{\circ} \mathrm{C}$ for $5 \mathrm{~min}$. HPV types were then classified via a nylon membrane immobilized with 21 different type-specific probes.

\section{Statistical Analysis}

Analyses were done with SPSS version 19.0 for Windows and Excel (version 2011). Descriptive statistical analysis was performed on the distribution of HPV genotypes using indicators such as frequency and prevalence. The Chi square test was used to test the differences between prevalence, genotypes and number of co-infections of HPV in different age groups and time groups.

\section{Ethical Consideration}

The study was approved by the ethics Committee of Chenzhou N0.1 People's Hospital and conducted strictly in accordance with the Declaration of Helsinki, including the confidentiality and anonymity. No informed consent or other action on the part of the patients was required due to anonymous analyses of the data. All experiments were carried out in the lab certified by the National Center for Clinical Laboratories following the laboratory biosafety guidelines.

\section{Results}

\section{Overall prevalence and genotype distribution of HPV}

In the seven years of surveillance, 214,715 eligible female cases were enrolled for final statistical analysis in this study. Of the adequate specimens, the overall prevalence of HPV DNA was $18.71 \%(95 \% \mathrm{Cl}$, $18.55 \%-18.88 \%$ ) for all types (Table 1). From 2012 to 2018 , the rate was $21.63 \%(95 \% \mathrm{Cl}, 21.07 \%-22.20 \%)$ 
in 2012 , which declined to $16.80 \%(95 \% \mathrm{Cl}, 16.41 \%-17.20 \%)$ in 2016 and $18.75 \%(95 \% \mathrm{Cl}, 18.35 \%-19.16 \%)$ in 2018, showing a downward trend in general. The prevalence of single and multiple HPV infections, as well as HPV genotypes excluding HPV51, -39 and -53 also showed a similar decreasing pattern (Fig. 1A, B).

HPV52 (5.12\%; 95\% Cl, 5.03\%-5.21\%) was the most common genotype, followed by HPV16 (2.96\%; 95\% $\mathrm{Cl}, 2.89 \%-3.03 \%),-58$ (2.51\%; $95 \% \mathrm{Cl}, 2.44 \%-2.58 \%),-81$ (1.86\%; $95 \% \mathrm{Cl}, 1.80 \%-1.92 \%)$ and -53 (1.84\%; $95 \% \mathrm{Cl}, 1.78 \%-1.90 \%$ ), accounting for $76.36 \%$ of all detected HPV positive infection types (Table 1 ). In addition, HPV52, - 16 and - 58 were the top three prevalent genotypes among HR-HPV, and the counterparts of LR-HPV were HPV81, -6 and -11 respectively (Table 1 and Fig. 2).

\section{Prevalence of high-risk, low-risk HPV types and by age}

Age groups are usually categorized as less than 21 (the youngest group), $21-30,31-40,41-50,51-60$ and more than 60 years (the oldest group). The total prevalence of HPV was $33.11 \%(95 \% \mathrm{Cl}$, $31.13 \%-35.15 \%)$ in women aged less than 21 years, $20.07 \%(95 \% \mathrm{Cl}, 19.70 \%-20.44 \%)$ in women aged $21-$ 30 years, $17.29 \%(95 \% \mathrm{Cl}, 17.01 \%-17.57 \%)$ in women aged $31-40$ years, $17.23 \%(95 \% \mathrm{Cl}, 16.95 \%-17.21 \%)$ aged in women $41-50$ years, $21.65 \%(95 \% \mathrm{Cl}, 21.11 \%-22.20 \%)$ in women aged $51-60$ years, and $25.95 \%$ (95\% Cl, 24.86\%-27.07\%) in women aged the oldest group. Age-specific HPV distribution showed a bimodal pattern. Two peaks of HPV infection were detected in the population of the youngest group (33.11\%) and the oldest group (25.95\%) (Table 3), showing young women are more prone to HPV infection. The overall HR-HPV and LR-HPV positive rate was $20.63 \%$ (95\% Cl 20.46-21.80\%), 3.67\% (95\% $\mathrm{Cl} 3.59-3.75 \%$ ) respectively. We also found the prevalence of HR-HPV infection was significantly higher among women with single infection (12.46\%) than those with multiple HPV infections $(8.17 \%, P<0.001)$, and a similar result was also observed for LR- HPV infection ( $2.0 \%$ vs. $1.67 \%, P<0.001$, Table 1$)$.

As shown in Table 2, the prevalence of HR-HPV only, LR-HPV only, and mixed LR- and HR-HPV infections were 15.17\% (95\% Cl, 15.02\%-15.32\%), 2.07\% (95\% Cl, 2.01\%-2.13\%), 1.47\% (95\% Cl, 1.42\%-1.52\%) respectively, and a significant difference in the distribution of HR-HPV only, LR-HPV only, and mixed LRand HR-HPV infections was observed among the six age groups. The highest prevalence of HR-HPV only infection (20.04\%) was found in the youngest age group, which decreased gradually as the age increased (21-30, 31-40 and $41-50$ years) before another increasing trend in the older age groups $(17.53 \%$ for the group of $51-60$ years old and $20.70 \%$ for women $>60$ years old), showing a bimodal curve. A similar agespecific prevalence pattern was also noted in LR-HPV only, and mixed LR-and HR-HPV infections.

\section{Prevalence of single and multiple HPV infections}

The prevalence of single HPV infection was $14.46 \%$ (14.46\%; $95 \% \mathrm{Cl}, 14.31 \%-14.61 \%)$ accounting for $77.30 \%$ of the $40,168 \mathrm{HPV}$-positive cases, whereas multiple infections $(4.25 \%$; $95 \% \mathrm{Cl}, 4.17 \%-4.34 \%)$ were the rare infection form. For participants infected with multiple HPV genotypes, the prevalence of HPV 
decreased significantly as the number of infected genotypes increased, with $6,926(3.23 \% ; 95 \% \mathrm{Cl}$, $3.16 \%-3.31 \%)$ cases of double infection, $1,589(0.74 \% ; 95 \% \mathrm{Cl}, 0.70 \%-0.78 \%)$ cases of triple infection, 427 $(0.20 \% ; 95 \% \mathrm{Cl}, 0.18 \%-0.22 \%)$ cases of quadruple infection and $178(0.08 \% ; 95 \% \mathrm{Cl}, 0.07 \%-0.09 \%)$ cases of more than five HPV genotype infections (Fig. 2 and Table 3). Notably, four of them were found to be infected with nine HPV types (data not shown).

Among the six age groups, there were significant differences in the distribution of single and multiple HPV infections $(P<0.001$, Table 2). Young women aged of less than 21 years had the highest prevalence of both single (19.47\%) and multiple HPV infection (13.64\%), followed by women aged of more than 60 years $(17.12 \%$ vs. $8.83 \%)$ and $51-60$ years $(15.89 \%$ vs. $5.76 \%)$ respectively, further confirmed our previous finding of a high susceptibility to HPV infection in the young population.

\section{Prevalence of LR-HPV only, HR-HPV only, and Mixed LR- and HR-HPV infections by number of HPV genotypes}

As shown in Table 3, there was significant different distributions of LR-HPV only $(P<0.001)$, HR-HPV only $(P<0.001)$, and Mixed LR- and HR-HPV infections $(P<0.001)$ among women co-infected with a different number of HPV genotypes. HR-HPV only infection $(86.17 \%, 26,755 / 31,048)$ still took up the majority in women with single HPV infection, as well as in the other group of multiple HPV infections. Both of the LR and HR-HPV only infection had the highest prevalence in single HPV infection $(2.00 \%$ vs. $12.46 \%$, respectively), which declined gradually as the number of co-infections increased. For individuals with multiple HR-HPV only infection, the frequencies of $\geq 5,4,3$, and 2 genotypes were $0.02 \%, 0.08 \%, 0.40 \%$, and $2.21 \%$, respectively. Especially, HR-HPV only infection was more frequently observed in those with double and triple HPV infection, and mixed LR- and HR-HPV infections in those with more than triple HPV infections, while none of these triple or more HPV genotypes have been detected in those with LR-HPV only infections. These results indicated that LR-HPV only infection primarily occurred in women with single HPV infection, and HR-HPV infections in multiple HPV infections.

\section{Discussion}

Cervical cancer burden occurs more often in Southern China where had been classified as "less developed region", hence special attention should be paid to the primary prevention in the region. Here we measured the prevalence of 21 distinct HPV types prior to widespread HPV vaccination among 214,715 women. The large sample sizes enable precise estimates of both increases and decreases in HPV type specific prevalence, which could be used as a baseline for comparison to future samplings of the population. The overall prevalence of HPV DNA in this representative sample of women was $18.71 \%$, with the highest prevalence (33.11\%) among women aged less than 21 years, corresponding with the data reported by Curacao (19.7\%) [15]. Global HPV prevalence estimates are well-known to vary by the region, study design, target population and calendar time [16]. HPV positive rates range from $6.70-44.50 \%$ in China, 
Especially, Western Asia (1.70\%) and North America (4.70\%) had a low rates of HPV infection, while East Africa $(33.6 \%)$ and the Caribbean (35.4\%) had a high rates of HPV infection [17-19].

In our study, HPV prevalence showed a significant downward trend, which declined obviously from 2012 to $2016(21.6 \%$ \& $16.8 \%)$. Analogous results of the HR-HPV positive rates were also found to decline from $25.3 \%$ in 2007 to $18.4 \%$ in 2014 , in Guangzhou [20]. This decline may be caused probably by the following reasons: 1) Emphasis on cervical cancer has led to increase participation in screening, including among women without cervical abnormalities; 2 ) In the last decade, some women that tested positive for HPV, due to immunization and treatment, became negative; and 3) Individuals were more likely to be aware of HPV and HPV vaccination following the continuous improvements of living conditions and health, which also led to the current rate of decline. Several studies have demonstrated the association of HPV with economic development. Women from impoverished countries and areas have suffered a high prevalence of HPV, e.g., $66.7 \%$ among young females in South Africa and $44.5 \%$ in Henan province of China $[19,21]$. However, a low rate of $6.7 \%$ was found among women in Beijing-the capital of China, which remained the most flourishing cultural and economic center in China, and also had the excellent healthcare system, indicating a strong association of HPV with socioeconomic development [22]. Additionally, the prevalence of HPV increased to $18.75 \%$ in the next two year may be partially explained by the alternation of high risk sexual behaviors including sexual openness, premarital sexual permissiveness and sex addiction, and the accuracy and sensitivity of testing assays [23, 24].

HPV genotype distribution in Asia was heterogeneous, which was probably due to the broad geographical and cultural diversity [25]. Several studies have revealed the overall prevalence of HR-HPV ranging from $9.90-27.50 \%$ in China, which is $20.63 \%$ in current study, as with that in Guangdong (20.02\%), Guiyang (20.45\%) and Nanning (22.28\%) [26]. In addition, we found HPV52 was the most commonly detected genotype, in agreement with that stated in Japan, Taiwan, and eastern Africa [27], however inconsistent with the data reported that HPV16 was the predominant genotype in other studies [3] and HPV35 in subSaharan Africa [28]. Moreover, prevalence of both HPV52 (5.12\%) and - 58 (2.51\%) was higher than that reported (2.3\% and $1.0 \%$ respectively) in the United States [3], and that from a nationwide populationbased investigation in 37 cities in China [26]. A large number of studies have demonstrated HPV 16 to be the most prevalent type, although its prevalence varied based on the population evaluated. We found the overall prevalence of HPV-16 to be low, which was in accordance with an estimated HPV16 prevalence of $3.2 \%$ from a meta-analysis of 1 million women with normal cytology [29]. Interestingly, HPV 16 is the strain most likely to cause cancer. Beyond that, clinic-based studies usually have found higher prevalence of HPV16 than population-based studies have $[27,30]$. It is possible that most women from the physical examination center enrolled in our study were more likely to detect HPV types not related to cervical infection. Apart from HPV16, -18-a common genotype of cervical cancer-was relatively low compared to the data performed by Chen [5]. Our data also indicated that HPV52, -16, and - 58 were consistently the top three HR-HPV genotypes from 2012 to 2018, suggesting the HPV vaccine covering these HR-HPV types is routinely recommended, especially for those females at a young age exposed to HPV. 
A two-variable analysis of HR-HPV infection-related variables showed a statistically significant association for age, number of sexual partners, hormonal contraception use and smoking [28]. The trend here in HR-HPV infection exhibiting elevated rates in younger groups and low rates in middle age groups reflected the natural history of HPV infection. Evaluations in the United States also showed that young women had the highest HPV prevalence [3], as well as the findings of the highest HPV prevalence (33.11\%) among women aged less than 21 years in our study. Young women often have a high infection rate, mainly because they are more sexually active before their immune systems become less sensitive [17]. Besides, the prevalence of HPV slightly declined in middle age, yet significantly increased among the oldest people, which are consistent with those in most developed countries and the data on Bruni and colleagues [29]. The mechanism of this increase in infection rates is unclear at present. Other than persistent infections seeming to be more prominent among female with such ages [19], this increase could be also explained by re-marriage, reactivation of latent HPV in menopausal women and the cohort variation [16].

Multiple HPV genotype infections were detected in approximately $22 \%$ of all positive cases in our study, consistent with that of Shanxi $(24.30 \%)$ and international populations [29], yet lower than that of Shanghai (36.60\%) [5]. In an unweighted analysis of these women, we found that most were multiple infections occurred either in young women aged less than 21 or those older than 51, and HR-HPV genotypes accounted for over $80 \%$ of multiple infections. Young women were known to have a high risk of HPV infection, however it is temporary and supposed to disappear within a year or two, thus which declined gradually by the increase of age [18]. Some have suggested HPV infection with multiple genotypes may prolong the duration of infection and increase the risk of cervical cancer and cervical precancerous lesions [31], result in a high prevalence of multiple infections among older women. Multiple infections were considered potentially to have competitive and/or cooperative interactions between HPV genotypes [32], although the mechanisms and potential oncogenic effects of multiple genotype infections warrants still need further investigation. Anyway such data could be beneficial to the development of HPV prophylactic vaccines.

The overall strengths of this study included the large sample size, PCR testing rather than serologic tests which allowed accurate determination of simultaneous co-infection, and the study was a decade long to measure a yearly trend of HPV infection. However, several limitations exist in our study. First, HPV DNA testing can't totally reflect the cumulative incidence of HPV and previous infection, which only represent current infection; second, our study included numerous specimens from women, not men, without pathological data, for example, cervical cytology and histology results, which was unable to explain the relationship between HPV infection and pathology, and unable to reflect the infection of HPV in general population in the region; and third, the detailed information about the patients, such as education level, economic status and background related to HPV infection, were not documented in this study, hindering a more comprehensive evaluation of the effects of these different backgrounds on the prevalence of HPV infection. 
In conclusion, we report the overall rate of HPV was $18.71 \%$, which showed a downward trend from $21.63 \%$ in 2012 to $18.75 \%$ in 2018 . Women aged less than 21 years had the highest HPV prevalence, who were unvaccinated suggests herd protection. Importantly, HPV52, -16, and - 58 were always the major genotypes, regardless of the fact that the rank varied according to the age and year. Such data would provide guidance for clinical care and public health policy including cervical screening and vaccination in Southern China, and would also be useful for the other low- and middle-income areas with a heavy HPV infection burden, to fight against cervical cancer.

\section{Abbreviations}

HPV: Human papillomavirus; HR-HPV: High-risk HPV; LR-HPV: Low- risk HPV; Cl『Confidence interval

\section{Declarations}

\section{Acknowledgements}

The authors would like to thank all the participants, as well as investigators and their study-site personnel, for their contribution to the study. We also thank Dr. Chenxu Guo (The Johns Hopkins University, School of Medicine) for the careful reading of the manuscript and Jian Zhang (Chenzhou N0.1 People's Hospital) for assistance with statistical analysis.

\section{Funding}

This work was supported by Hunan Science and Technology innovation project (2018SK50309), Hunan Health Commission project (B2019002) and Chenzhou Science and technology Bureau (yfzx201908, zdyf201941 and zdyf201947).

\section{Availability of data and materials}

All data and materials are included in the manuscript and thus available to the reader.

\section{Authors' contributions}

LP, ZY and HL drafted the manuscript and participated in the design of the study. $L P, H L, Z Y$ and XG performed the statistical analysis. Ping, QT, YH, YN, QZ, ST, Fan, Hua, and Qiu carried out the sample collection and HPV detection. All authors made substantive intellectual contributions, performed research, and read and approved the final manuscript.

\section{Ethics approval and consent to participate}

The study was approved by the ethics Committee of Chenzhou N0.1 People's Hospital. No informed consent or other action on the part of the patients was required owing to anonymous analyses of the data. 
Consent for publication

Not applicable.

Competing interests

The authors declare that they have no competing interests.

\section{Author details}

1 The First School of Clinical Medicine, Southern Medical University, Chenzhou NO.1 People's Hospital, Chenzhou 423000, P.R. China

2 Institute of Pathogenic Biology, Hengyang Medical College, Hunan Provincial Key Laboratory for Special Pathogens Prevention and Control, Hunan Province Cooperative Innovation Center for Molecular Target New Drug Study, University of South China, Hengyang 421001, P.R. China

\section{References}

1. Senkomago V, Henley SJ, Thomas CC, Mix JM, Markowitz LE, Saraiya M: Human PapillomavirusAttributable Cancers - United States, 2012-2016. MMWR Morb Mortal Wkly Rep 2019; 68(33):724728.

2. Siegel RL, Miller KD, Jemal A: Cancer statistics, 2015. CA Cancer J Clin 2015; 65(1):5-29.

3. Dunne EF, Unger ER, Sternberg M, McQuillan G, Swan DC, Patel SS, Markowitz LE: Prevalence of HPV infection among females in the United States. JAMA 2007; 297(8):813-819.

4. Chen $W$, Zheng $R$, Zeng $H$, Zhang S: The incidence and mortality of major cancers in China, 2012. Chin J Cancer 2016; 35(1):73.

5. Oliver SE, Unger ER, Lewis R, McDaniel D, Gargano JW, Steinau M, Markowitz LE: Prevalence of Human Papillomavirus Among Females After Vaccine Introduction-National Health and Nutrition Examination Survey, United States, 2003-2014. J Infect Dis 2017; 216(5):594-603.

6. Bellaminutti S, Seraceni S, De Seta F, Gheit T, Tommasino M, Comar M: HPV and Chlamydia trachomatis co-detection in young asymptomatic women from high incidence area for cervical cancer. J Med Viro/ 2014; 86(11):1920-1925.

7. Aro K, Nieminen P, Louvanto K, Jakobsson M, Virtanen S, Lehtinen M, Dillner J, Kalliala I: Age-specific HPV type distribution in high-grade cervical disease in screened and unvaccinated women. Gynecol Oncol 2019; 154(2):354-359.

8. Lee NR, Winer RL, Cherne S, Noonan CJ, Nelson L, Gonzales AA, Umans JG, Buchwald D, Collaborative to Improve Native Cancer O: Human Papillomavirus Prevalence Among American Indian Women of the Great Plains. J Infect Dis 2019; 219(6):908-915.

9. Wang Y, Meng Y, Li W, Zhang X, Deng Z, Hu M, Shen P, Xu S, Fu C, Jiang W et al: Prevalence and Characteristics of hrHPV Infection among 414,540 Women: A Multicenter Study in Central and 
Eastern China. J Cancer 2019; 10(8):1902-1908.

10. Ferre VM, Ekouevi DK, Gbeasor-Komlanvi FA, Collin G, Le Hingrat Q, Tchounga B, Salou M, Descamps D, Charpentier C, Dagnra AC: Prevalence of human papillomavirus, human immunodeficiency virus and other sexually transmitted infections among female sex workers in Togo: a national crosssectional survey. Clin Microbiol Infect 2019; 25(12):1560 e1561-1560 e1567.

11. Li K, Li Q, Song L, Wang D, Yin R: The distribution and prevalence of human papillomavirus in women in mainland China. Cancer 2019; 125(7):1030-1037.

12. Owusu-Edusei K, Jr., Chesson HW, Gift TL, Tao G, Mahajan R, Ocfemia MC, Kent CK: The estimated direct medical cost of selected sexually transmitted infections in the United States, 2008. Sex Transm Dis 2013; 40(3):197-201.

13. Chow EP, Danielewski JA, Fehler G, Tabrizi SN, Law MG, Bradshaw CS, Garland SM, Chen MY, Fairley CK: Human papillomavirus in young women with Chlamydia trachomatis infection 7 years after the Australian human papillomavirus vaccination programme: a cross-sectional study. Lancet Infect Dis 2015; 15(11):1314-1323.

14. Braaten KP, Laufer MR: Human Papillomavirus (HPV), HPV-Related Disease, and the HPV Vaccine. Rev Obstet Gynecol 2008; 1(1):2-10.

15. Hooi DJ, Lissenberg-Witte BI, Kenter G, de Koning MNC, Gomes Bravio I, Ardts K, Kleinmoedig S, Benita E, Pinedo HM, Berkhof $\mathrm{J}$ et al: Human papillomavirus (HPV) prevalence and associated risk factors in women from Curacao. PLoS One 2018; 13(7):e0199624.

16. Brotherton JM, Hawkes D, Sultana F, Malloy MJ, Machalek DA, Smith MA, Garland SM, Saville M: Age-specific HPV prevalence among 116,052 women in Australia's renewed cervical screening program: A new tool for monitoring vaccine impact. Vaccine 2019; 37(3):412-416.

17. Jiang L, Tian X, Peng D, Zhang L, Xie F, Bi C, Wang R, Wang J, Qi D: HPV prevalence and genotype distribution among women in Shandong Province, China: Analysis of 94,489 HPV genotyping results from Shandong's largest independent pathology laboratory. PLoS One 2019; 14(1):e0210311.

18. Wang J, Tang D, Wang K, Wang J, Zhang Z, Chen Y, Zhang X, Ma C: HPV genotype prevalence and distribution during 2009-2018 in Xinjiang, China: baseline surveys prior to mass HPV vaccination. BMC Womens Health 2019; 19(1):90.

19. Zhao R, Zhang WY, Wu MH, Zhang SW, Pan J, Zhu L, Zhang YP, Li H, Gu YS, Liu XZ: Human papillomavirus infection in Beijing, People's Republic of China: a population-based study. $\mathrm{Br} \mathrm{J} C a n c e r$ 2009; 101(9):1635-1640.

20. Chen X, Xu H, Xu W, Zeng W, Liu J, Wu Q, Zhao X, Jiang T: Prevalence and genotype distribution of human papillomavirus in 961,029 screening tests in southeastern China (Zhejiang Province) between 2011 and 2015. Sci Rep 2017; 7(1):14813.

21. Mbulawa ZZA, van Schalkwyk C, Hu NC, Meiring TL, Barnabas S, Dabee S, Jaspan H, Kriek JM, Jaumdally SZ, Muller E et al: High human papillomavirus (HPV) prevalence in South African adolescents and young women encourages expanded HPV vaccination campaigns. PLoS One 2018; 13(1):e0190166. 
22. Thompson EL, Wheldon CW, Rosen BL, Maness SB, Kasting ML, Massey PM: Awareness and knowledge of HPV and HPV vaccination among adults ages 27-45 years. Vaccine 2020; 38(15):31433148.

23. Wigfall L, Rawls A, Sebastian N, Messersmith A, Pirisi-Creek L, Spiryda L, Williams EM, Creek K, Glover SH: HPV high risk and protective behaviors: the effects of religious affiliation. $J$ Relig Health 2012; 51(4):1325-1336.

24. Pesic A, Krings A, Schreckenberger C, Hempel M, Preyer R, Kaufmann AM: Analytical Evaluation of the Human Papillomavirus HPV DNA Array E1-Based Genotyping Assay. Intervirology 2019; 62(34):124-133.

25. Clifford GM, Gallus S, Herrero R, Munoz N, Snijders PJ, Vaccarella S, Anh PT, Ferreccio C, Hieu NT, Matos $\mathrm{E}$ et al: Worldwide distribution of human papillomavirus types in cytologically normal women in the International Agency for Research on Cancer HPV prevalence surveys: a pooled analysis. Lancet 2005; 366(9490):991-998.

26. Wang R, Guo XL, Wisman GB, Schuuring E, Wang WF, Zeng ZY, Zhu H, Wu SW: Nationwide prevalence of human papillomavirus infection and viral genotype distribution in $\mathbf{3 7}$ cities in China. BMC Infect Dis 2015; 15:257.

27. de Sanjose S, Diaz M, Castellsague X, Clifford G, Bruni L, Munoz N, Bosch FX: Worldwide prevalence and genotype distribution of cervical human papillomavirus DNA in women with normal cytology: a meta-analysis. Lancet Infect Dis 2007; 7(7):453-459.

28. Torres-Poveda K, Ruiz-Fraga I, Madrid-Marina V, Chavez M, Richardson V: High risk HPV infection prevalence and associated cofactors: a population-based study in female ISSSTE beneficiaries attending the HPV screening and early detection of cervical cancer program. BMC Cancer 2019; 19(1):1205.

29. Bruni L, Diaz M, Castellsague X, Ferrer E, Bosch FX, de Sanjose S: Cervical human papillomavirus prevalence in 5 continents: meta-analysis of 1 million women with normal cytological findings. $J$ Infect Dis 2010; 202(12):1789-1799.

30. Castle PE, Schiffman M, Bratti MC, Hildesheim A, Herrero R, Hutchinson ML, Rodriguez AC, Wacholder S, Sherman ME, Kendall $\mathrm{H}$ et al: A population-based study of vaginal human papillomavirus infection in hysterectomized women. J Infect Dis 2004; 190(3):458-467.

31. Schmitt M, Depuydt C, Benoy I, Bogers J, Antoine J, Arbyn M, Pawlita M, Group VS: Multiple human papillomavirus infections with high viral loads are associated with cervical lesions but do not differentiate grades of cervical abnormalities. J Clin Microbio/ 2013; 51(5):1458-1464.

32. Senapati R, Nayak B, Kar SK, Dwibedi B: HPV genotypes co-infections associated with cervical carcinoma: Special focus on phylogenetically related and non-vaccine targeted genotypes. PLoS One 2017; 12(11):e0187844.

\section{Tables}

Table 1. Overall prevalence of HPV genotype in single and multiple infections. 


\begin{tabular}{|c|c|c|c|c|c|c|}
\hline \multirow[t]{2}{*}{ genotypes } & \multicolumn{2}{|c|}{ single infection } & \multicolumn{2}{|c|}{ multiple infections } & \multicolumn{2}{|c|}{ Total } \\
\hline & $(n, \%)$ & $95 \% \mathrm{CI}$ & $(n, \%)$ & $95 \% \mathrm{CI}$ & $(n, \%)$ & $95 \% \mathrm{CI}$ \\
\hline \multicolumn{7}{|l|}{ HR-HPV* } \\
\hline 52 & $7,457(3.47)$ & 3.39-3.55 & 3,537(1.65) & $1.60-1.70$ & $10,994(5.12)$ & $5.03-5.21$ \\
\hline 16 & $4,183(1.95)$ & $1.89-2.01$ & $2,175(1.01)$ & $0.97-1.05$ & $6,358(2.96)$ & $2.89-3.03$ \\
\hline 58 & $3,267(1.52)$ & $1.47-1.57$ & $2,128(0.99)$ & $0.95-1.03$ & $5,395(2.51)$ & $2.44-2.58$ \\
\hline 53 & $2,261(1.05)$ & $1.01-1.09$ & 1,683(0.78) & $0.74-0.82$ & $3,944(1.84)$ & $1.78-1.90$ \\
\hline 39 & $1,747(0.81)$ & $0.77-0.85$ & $1,330(0.62)$ & $0.59-0.65$ & $3,077(1.43)$ & $1.38-1.48$ \\
\hline 51 & $1,284(0.60)$ & $0.57-0.63$ & $1,030(0.48)$ & $0.45-0.51$ & $2,314(1.08)$ & $1.04-1.12$ \\
\hline 68 & $1,187(0.55)$ & $0.52-0.58$ & $1,092(0.51)$ & $0.48-0.54$ & $2,279(1.06)$ & $1.02-1.10$ \\
\hline 33 & $1,231(0.57)$ & $0.54-0.60$ & $1,031(0.48)$ & $0.45-0.51$ & $2,262(1.05)$ & $1.01-1.09$ \\
\hline 18 & $1,279(0.60)$ & $0.57-0.63$ & $878(0.41)$ & $0.38-0.44$ & $2,157(1.00)$ & $0.96-1.04$ \\
\hline 31 & $841(0.39)$ & $0.36-0.42$ & $664(0.31)$ & $0.29-0.33$ & $1,505(0.70)$ & $0.67-0.74$ \\
\hline 66 & $598(0.28)$ & $0.26-0.30$ & $639(0.30)$ & $0.28-0.32$ & $1,237(0.58)$ & $0.55-0.61$ \\
\hline 56 & $501(0.23)$ & $0.21-0.25$ & $520(0.24)$ & $0.22-0.26$ & $1,021(0.48)$ & $0.45-0.51$ \\
\hline 59 & $436(0.20)$ & $0.18-0.22$ & $368(0.17)$ & $0.15-0.19$ & $804(0.37)$ & $0.35-0.40$ \\
\hline 45 & $225(0.10)$ & $0.09-0.11$ & $258(0.10)$ & $0.11-0.14$ & $483(0.22)$ & $0.20-0.24$ \\
\hline 35 & $258(0.12)$ & $0.11-0.14$ & $200(0.09)$ & $0.08-0.10$ & $458(0.21)$ & $0.19-0.23$ \\
\hline \multicolumn{7}{|l|}{ LR- HPV \# } \\
\hline 81 & $2,240(1.04)$ & $1.00-1.08$ & $1,743(0.81)$ & 0.77-0.85 & $3,983(1.86)$ & $1.80-1.92$ \\
\hline 6 & $765(0.36)$ & $0.34-0.39$ & $723(0.34)$ & $0.32-0.37$ & $1,488(0.69)$ & $0.66-0.73$ \\
\hline 11 & $643(0.30)$ & $0.28-0.32$ & $519(0.24)$ & $0.22-0.26$ & $1,162(0.54)$ & $0.51-0.57$ \\
\hline 44 & $380(0.18)$ & $0.16-0.20$ & $342(0.16)$ & $0.14-0.18$ & $722(0.34)$ & $0.32-0.37$ \\
\hline 42 & $173(0.08)$ & 0.07-0.09 & $148(0.07)$ & $0.06-0.08$ & $321(0.15)$ & $0.13-0.17$ \\
\hline 43 & $92(0.04)$ & 0.03-0.05 & $105(0.05)$ & $0.04-0.06$ & 197(0.09) & $0.08-0.10$ \\
\hline Total & $31,048(14.46)$ & 14.31-14.61 & $9,120(4.25)$ & $4.17-4.34$ & $40,168(18.71)$ & $18.55-18.88$ \\
\hline
\end{tabular}

single infection versus multiple infections considering HR-HPV $* P<0.001$ and LR- HPV ${ }^{\#} P<0.001$. HPV, human papillomavirus; HR-HPV, high-risk HPV; LR-HPV, low-risk HPV; CI, confidence interval.

Table 2. Age-specific distribution of HPV infection.

HPV, human papillomavirus; HR-HPV, high-risk HPV; LR-HPV, low-risk HPV; CI, confidence interval.

Table 3. Prevalence of LR-HPV only, HR-HPV only, and Mixed LR- and HR-HPV infections in women co-infection with different numbers.

\begin{tabular}{lcccccccc}
\hline \multirow{2}{*}{ number of co-infections } & \multicolumn{2}{c}{ LR-HPV only } & \multicolumn{2}{c}{ HR-HPV only } & \multicolumn{2}{c}{ mixed LR- and HR-HPV } & \multicolumn{2}{c}{ Total } \\
\cline { 2 - 9 } & n, (\%) & $95 \%$ CI & n, (\%) & $95 \%$ CI & n, (\%) & $95 \%$ CI & n, (\%) & $95 \%$ CI \\
\hline Total & $4,448(2.07)$ & $2.01-2.13$ & $32,571(15.17)$ & $15.02-15.32$ & $3,149(1.47)$ & $1.42-1.52$ & $40,168(18.71)$ & $18.55-18.88$ \\
Single HPV genotype & $4,293(2.00)$ & $1.94-2.06$ & $26,755(12.46)$ & $12.32-12.60$ & NA & NA & $31,048(14.46)$ & $14.31-14.61$ \\
Double HPV genotypes & $152(0.07)$ & $0.06-0.08$ & $4,752(2.21)$ & $2.15-2.27$ & $2,022(0.94)$ & $0.90-0.98$ & $6,926(3.23)$ & $3.16-3.31$ \\
Triple HPV genotypes & $3(0.00)$ & NA & $849(0.40)$ & $0.37-0.43$ & $737(0.34)$ & $0.32-0.37$ & $1,589(0.74)$ & $0.70-0.78$ \\
Quadruple HPV genotypes & $0(0.00)$ & NA & $169(0.08)$ & $0.07-0.09$ & $258(0.12)$ & $0.11-0.14$ & $427(0.20)$ & $0.18-0.22$ \\
$\geq$ Five HPV genotypes & $0(0.00)$ & NA & $46(0.02)$ & $0.01-0.03$ & $132(0.06)$ & $0.05-0.07$ & $178(0.08)$ & $0.07-0.09$ \\
$P$ & $<0.001$ & $<0.001$ & $<0.001$ & & $<0.001$ & \\
\hline
\end{tabular}




\begin{tabular}{|c|c|c|c|c|c|c|c|c|c|}
\hline & & \multicolumn{7}{|c|}{ Age, years (n, \%) } & \multirow[t]{2}{*}{$P$} \\
\hline & & $\begin{array}{c}<21 \\
(\mathrm{n}=2,111)\end{array}$ & $\begin{array}{c}21-30 \\
(n=45,211)\end{array}$ & $\begin{array}{c}31-40 \\
(n=70,681)\end{array}$ & $\begin{array}{c}41-50 \\
(n=68,488)\end{array}$ & $\begin{array}{c}51-60 \\
(n=22,142)\end{array}$ & $\begin{array}{c}>60 \\
(\mathrm{n}=6,082)\end{array}$ & Total & \\
\hline $\begin{array}{l}\text { HPV } \\
\text { numbers }\end{array}$ & positive & 699(33.11) & $9,073(20.07)$ & $12,223(17.29)$ & $11,801(17.23)$ & $4,794(21.65)$ & $1,578(25.95)$ & $40,168(18.71)$ & $<0.001$ \\
\hline \multicolumn{10}{|c|}{ Number of co-infections } \\
\hline $\begin{array}{r}\text { single } \\
\text { genotype }\end{array}$ & HPV & $411(19.47)$ & $6,818(15.08)$ & $9,795(13.86)$ & $9,464(13.82)$ & $3,519(15.89)$ & $1,041(17.12)$ & $31,048(14.16)$ & \multirow[t]{5}{*}{$<0.001$} \\
\hline $\begin{array}{c}\text { double } \\
\text { genotypes }\end{array}$ & HPV & $167(7.91)$ & $1,671(3.70)$ & $1,957(2.77)$ & $1,882(2.75)$ & $906(4.09)$ & $343(5.64)$ & $6,926(3.23)$ & \\
\hline $\begin{array}{c}\text { triple } \\
\text { genotypes }\end{array}$ & HPV & $74(3.51)$ & $438(0.97)$ & $349(0.49)$ & $350(0.51)$ & $257(1.16)$ & 121(1.99) & $1,589(0.74)$ & \\
\hline $\begin{array}{l}\text { Quadruple } \\
\text { genotypes }\end{array}$ & HPV & $32(1.52)$ & $108(0.24)$ & $90(0.13)$ & $78(0.11)$ & $74(0.33)$ & $45(0.74)$ & $427(0.20)$ & \\
\hline $\begin{array}{c}\quad \geq \text { five } \\
\text { genotypes }\end{array}$ & HPV & $15(0.71)$ & $38(0.08)$ & $32(0.05)$ & $27(0.04)$ & $38(0.17)$ & $28(0.46)$ & $178(0.08)$ & \\
\hline \multicolumn{10}{|l|}{ HPV genotype } \\
\hline HR-HPV only & & $423(20.04)$ & $7,122(15.75)$ & $10,146(14.35)$ & $9,742(14.22)$ & $3,881(17.53)$ & $1,259(20.70)$ & $32,573(15.17)$ & \multirow[t]{3}{*}{$<0.001$} \\
\hline LR-HPV only & & $114(5.40)$ & $1,123(2.48)$ & $1,309(1.85)$ & 1,293(1.89) & $484(2.19)$ & $125(2.06)$ & $4,448(2.07)$ & \\
\hline $\begin{aligned} & \text { mixed LR- } \\
& \text { HR-HPV }\end{aligned}$ & and & $162(7.67)$ & $828(1.83)$ & $768(1.09)$ & $766(1.12)$ & $429(1.94)$ & 194(3.19) & $3,147(1.47)$ & \\
\hline
\end{tabular}

HPV, human papillomavirus; HR-HPV, high-risk HPV; LR-HPV, low-risk HPV; CI, confidence interval. NA: Not applicable.

\section{Figures}

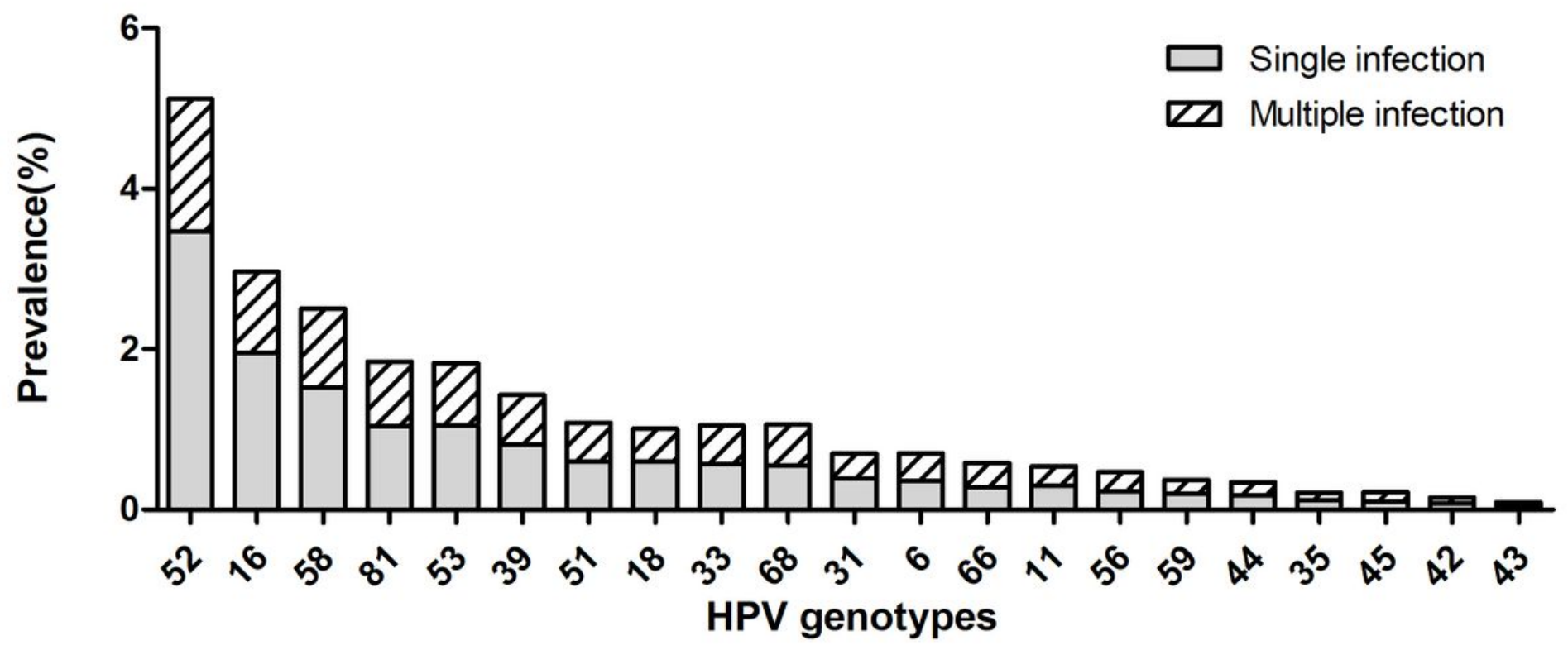

Figure 1 
Prevalence of HPV infections. A: Prevalence of HPV infections among females, 2012-2018; B: genotypespecific distribution of HPV infections, 2012-2018. The six top prevalent HPV genotypes were HPV52 (5.12\%), -16 (2.96\%), -58 (2.51\%), -53 (1.84\%), -39 (1.43\%), and -51 (1.08\%) respectively. HPV genotypes, except for HPV51, -39 and -53, presented a tendency of decrease from 2012-2018.
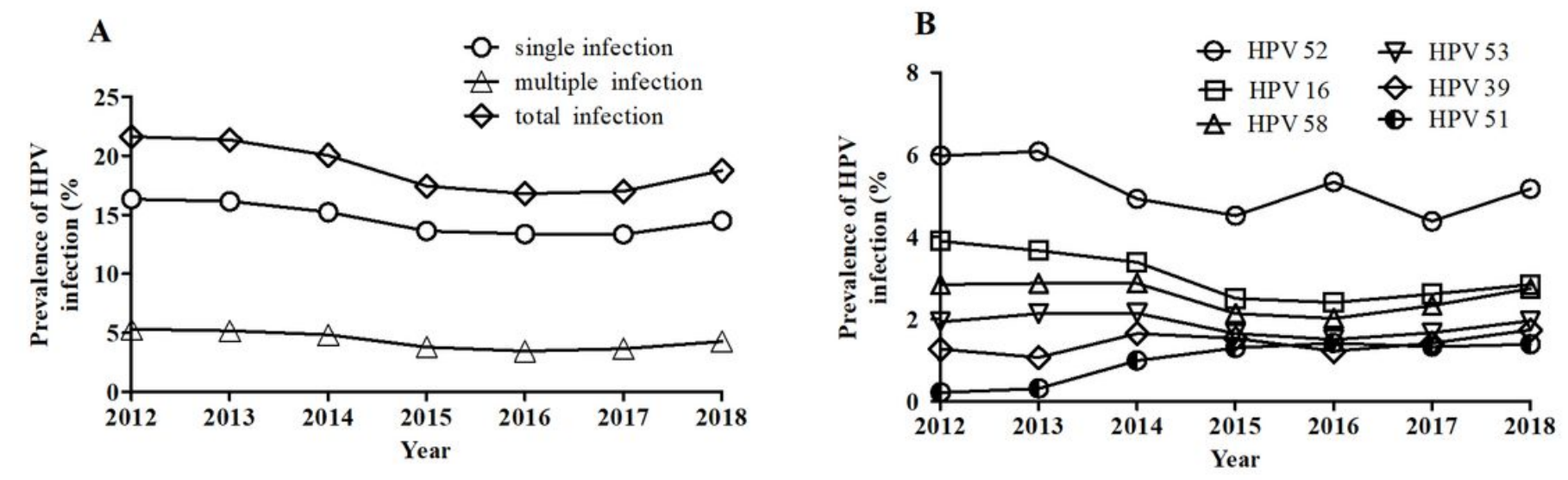

\section{Figure 2}

Prevalence of HPV genotypes in single and multiple infections. Fifteen HR-HPV genotypes: HPV52, -16, $-58,-53,-39,-51,-68,-33,-18,-31,-66,-56,-59,-45$ and -35 ; six LR-HPV genotypes: HPV81, $-6,-11,-44,-42$ and -43 . 